http://jmscr.igmpublication.org/home/ ISSN (e)-2347-176x ISSN (p) 2455-0450 crossref DOI: https://dx.doi.org/10.18535/jmscr/v8i4.17

\author{
(D) Journal Of Medical Science And Clinical Research \\ IGM Publication \\ An official Publication of IGM Publication
}

\title{
A Pictorial Essay of MRI Findings - Obstetric Brachial Plexopathy
}

Authors

\author{
Dr Dhruva B Shetty ${ }^{1}$, Dr Balakrishna Shetty ${ }^{2}$, Dr G Gurushankar ${ }^{3}$, Dr Anand S.H ${ }^{4}$ \\ ${ }^{1}$ Resident, Dept of Radiodiagnosis, Sri Siddhartha Medical College and Hospital Tumkur \\ ${ }^{2}$ Vice Chancellor, Sri Siddhartha Medical College and Hospital \\ ${ }^{3}$ Head of the Department, Dept of Radiodiagnosis, Sri Siddhartha Medical College and Hospital \\ ${ }^{4}$ Associate Professor, Dept of Radiodiagnosis, Sri Siddhartha Medical College and Hospital
}

\section{Learning Objectives}

Review of 25 patients with obstetric brachial plexus palsy and determine the effectiveness and spectrum of MRI findings in these infants, which will help in emphasizing on imaging techniques, subtypes of injuries and importance of early MRI.

\section{Background}

Brachial plexus palsy in neonates occurs when an infant's upper shoulder is obstructed by the pubic symphysis, nerve injury can occur anywhere along the brachial plexus. The most common site being supraclavicular part emanating varied neurologic deficits

Brachial plexopathies cannot be diagnosed accurately with clinical examination because of the complicated plexus design and also the types of lesions and injuries are frequently complex.

EMG provides functional involvement but lacks localization.

Improvements in the surgical field have made presurgical imaging a necessity.

The multiplanar, non-radiative and non invasive capabilities of MRI has made it the modality of choice

\section{Objective}

To determine the effectiveness and spectrum of MRI findings in infants with obstetric brachial plexus palsy, which will help in further management.

\section{Materials \& Methods Procedure Details}

25 patients clinically suspected with obstetrical brachial plexus palsy (1-48 months) underwent MRI of the brachial plexus

\section{Protocol}

MR images of the brachial plexus were performed on a 1.5T magnet and consisted of T1- and T2weighted sequences in the axial, sagittal and coronal planes, targeting both sides.

T1W FSE (TR/TE: 500/10, FOV:22-28 cm, matrix: $320 \times 224 \mathrm{~mm}$ ) in the axial oblique, coronal oblique and sagittal oblique planes.

T2W FSE sequence (TR/TE: 4000/110), FOV: 22-

$28 \mathrm{~cm}$, matrix: $384 \times 256 \mathrm{~mm}$ ) in the axial oblique plane

STIR Coronal: (TR/TE 4,000- 6,000/60-100; inversion time, 150 milliseconds; echo-train length, 10; field of view, 140-400 mm2; matrix 
size, 256-384 × 224-256; section thickness, 3-5 $\mathrm{mm}$; and intersection gap, 0.3-1.0 mm)

Axial plane - to show the anterior and posterior rootlets and the named roots exiting the neural foramina.

Coronal and sagittal plane-Trunks, divisions, cords, and branches are easily seen on coronal images, with the sagittal and axial planes used for problem solving. The trunks are positioned between the anterior and middle scalene muscles. Sagittal plane useful to identify subclavian artery medial, lateral, and posterior cords, as they are named according to their relation to this artery.

\section{MR Imaging Findings}

Injuries of the brachial plexus are classified into 3 categories

1. Preganglionic injury, representing nerve root avulsion.(fig 9)

2. Postganglionic injury, which signifies injury distal to the sensory root ganglion and is further classified into nerve rupture or lesion in continuity (neuropraxia).

3. Combination of pre and postganglionic injury.

\section{MRI Findings in Preganglionic Injury:}

- Root avulsion (fig 8)

- Pseudomeningocele (fig 6)

- Spinal cord edema at the level of root avulsion

- Spinal cord avulsion

- Displacement of the spinal cord to the avulsion site

MRI findings of preganglionic injury show avulsion of the nerve roots at their origin. Pseudomeningoceles can form; due to extravasation of cerebrospinal fluid (CSF) through a tear of the perineural sheath, seen as fluidintensity lesions at the site of nerve root avulsion on T2WI. In mild injury, T2 hyperintensity is noted only on STIR images without thickening MRI Findings in Postganglionic injury

MRI findings of post-ganglionic injury are variable. Neuropraxic injury is seen as thickened nerves or abnormal hyperintensity of the brachial plexus roots, trunks or cords on T2WI. Nerve ruptures are seen as discontinuity of neural structures with distal nerve contraction.

Associated denervation oedema within the affected muscles is a supportive finding of postganglionic injury (Fig. 7)

Lastly, MRI may demonstrate direct brachial plexus compression by a haematoma, fracture fragment or callus formation, which can also cause brachial plexopathy

Other findings included intra-spinal hematoma, paravertebral soft tissue contusion and shoulder dislocation (fig 11)

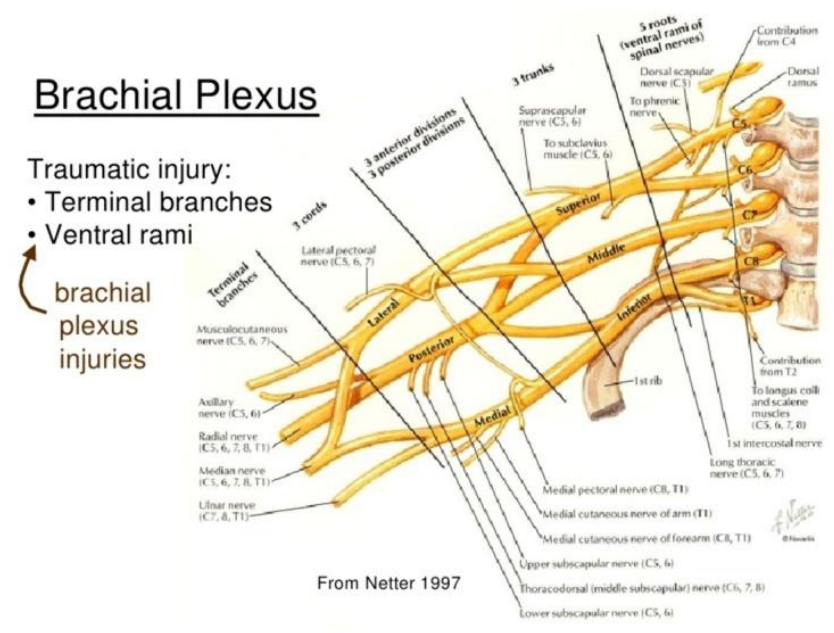

Fig. 1 Anatomy of the brachial plexus (C) Netters anatomy atlas

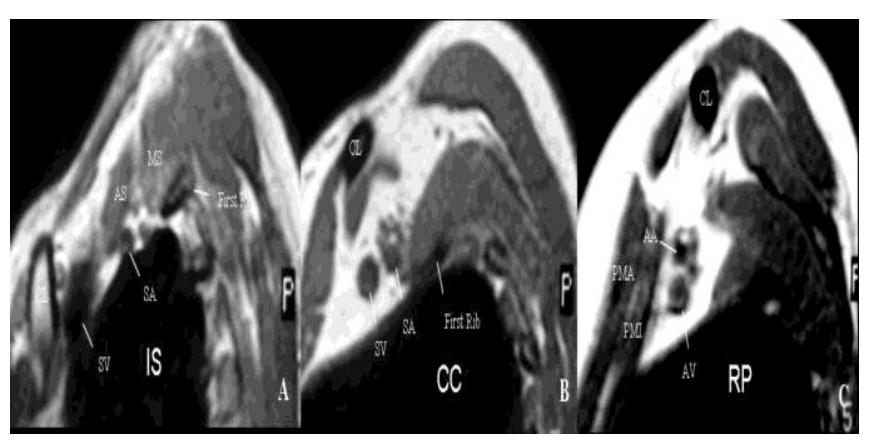

Fig. 2: Oblique sagittal T1 W MRI views (A, B, C from medial to lateral) demonstrate three parts of the BPL. Supraclavicular plexus is composed of roots and trunks. Roots are seen at the interscalene triangular space (IS) between anterior and middle scalene muscles. Subclavian artery forms the floor of the interscalene triangle (B). Roots then form the trunks at the lateral border of middle scalene 
muscles. Retroclavicular plexus is composed of divisions situated in the costoclavicular space (CC) between the first rib and clavicula and BPL is seen in superior and posterior aspect of the subclavican artery (C). Infraclavicular plexus is composed of cords and terminal branches located in the retropectoralis minor space (RP), BPL is situated in the posterior and superior aspect of axillary artery AA: axillary artery, ADs: anterior divisons, AS: anterior scalene muscle, AV:axillary vein, CL: clavicula, I:inferior trunk, LC:lateral cord, M:middle trunk, MC: medial cord, MS: middle scalene muscle, PC: posterior cord, PDs: posterior divisions, PMA: pectoralis major muscle, PMI: pectoralis minor muscle, $\mathrm{S}$ :superior trunk, SA: subclavian artery, SV: subclavian vein (C) Radiology, Akdeniz University - Antalya/TR

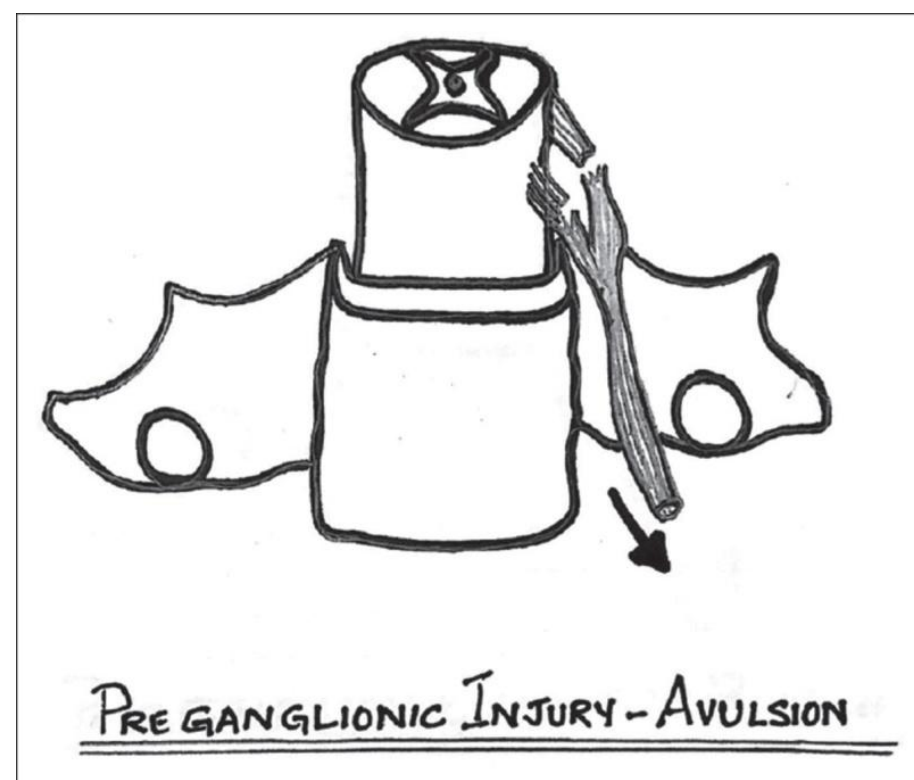

Fig. 3

(C) Dsouza AP, Tandon S, Gundogan M, Abdalla AA. Adding Value to the Magnetic

Resonance Examination in a Case of Brachial Plexus Palsy. J Clin Imaging Sci 2018;8:38

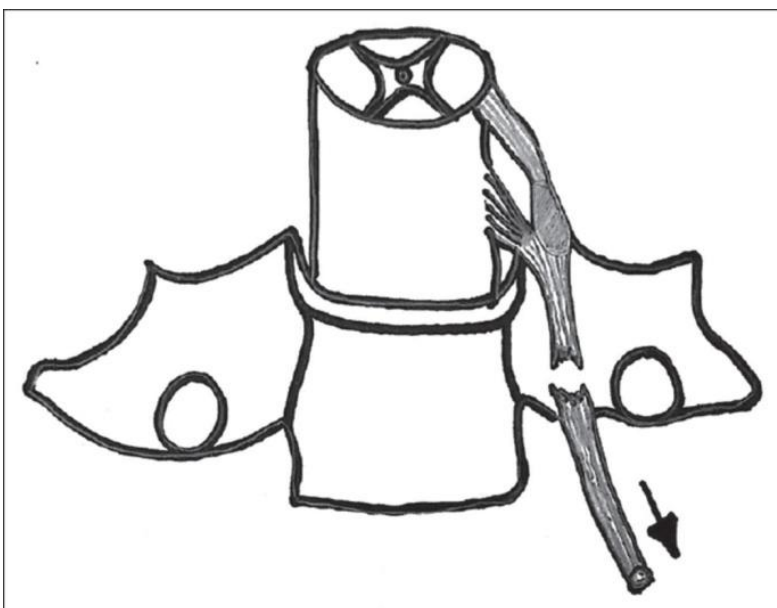

\section{Postganglionic Injury - Rupture}

Fig. 4

(C) Dsouza AP, Tandon S, Gundogan M, Abdalla AA. Adding Value to the Magnetic Resonance Examination in a Case of Brachial Plexus Palsy. J Clin Imaging Sci 2018;8:38

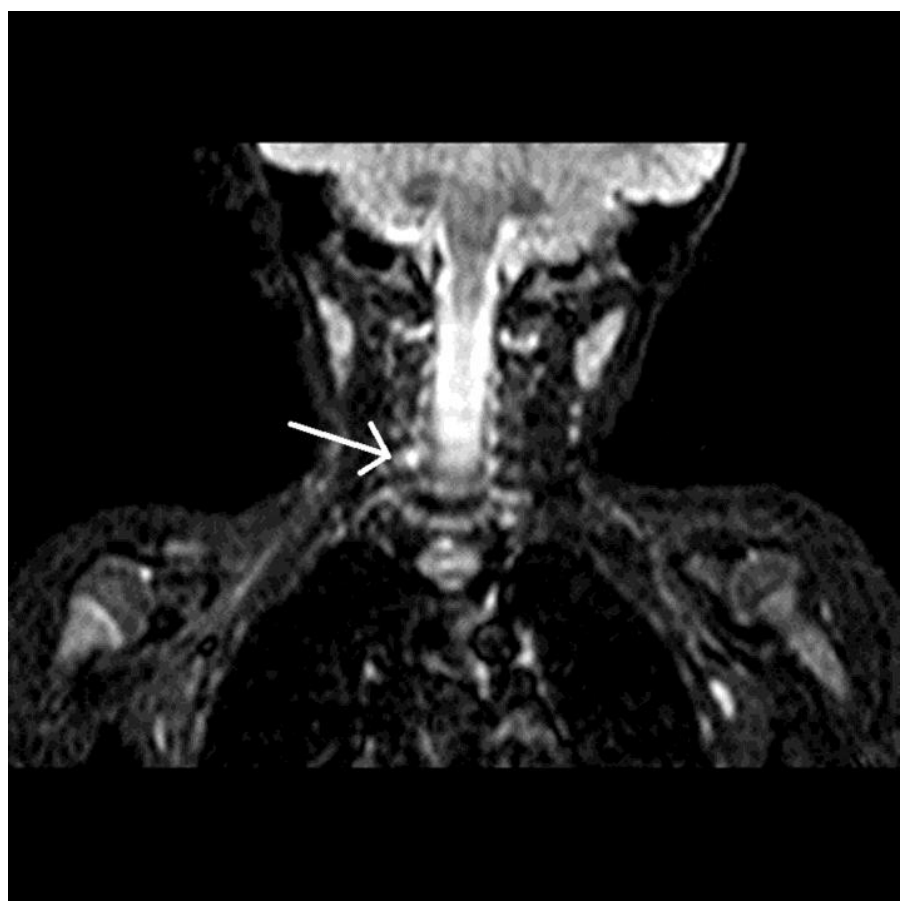

Fig. 5: 4 month old girl with clinically suspected brachial plexus palsy .coronal $\mathrm{t} 2$ image shows $\mathrm{T} 2$ hyperintensity at left C5-6 suggestive of root avulsion

(C) Sri Siddhartha academy of higher education, Sri Siddhartha medical college and hospital - Tumkur Karnataka/IN 


\section{JMSCR Vol||08||Issue||04||Page 89-93||April}

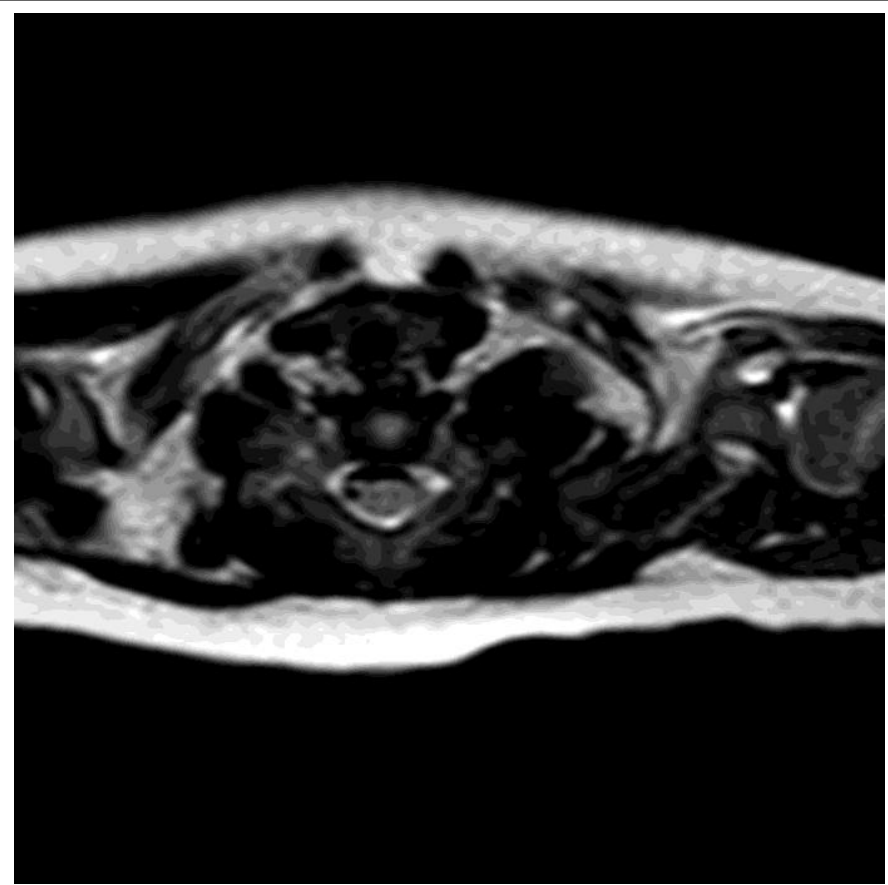

Fig. 6: A 11-month-old boy with clinically suspected left-sided brachial plexus palsy. Axial high-resolution MR imaging shows a pseudomeningocele at right C5-6 level. Note absent nerve roots on right side suggestive of nerve root avulsion injury.

(C) Sri Siddhartha academy of higher education, Sri Siddhartha medical college and hospital - Tumkur Karnataka/IN

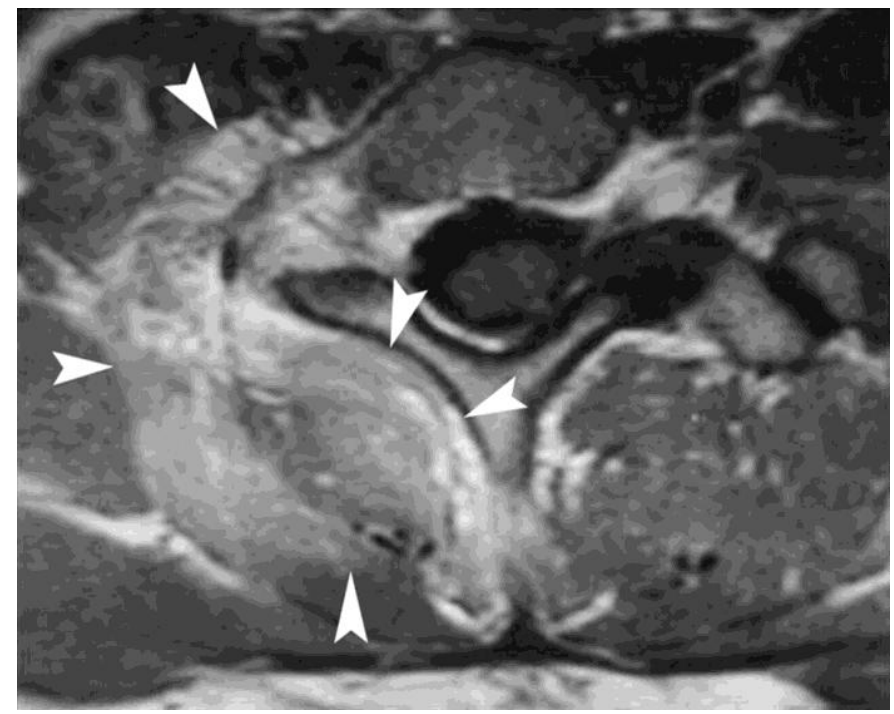

Fig. 7: Axial T2-weighted MR image demonstrates areas of hyperintensity (arrowheads) in the right paraspinal muscles

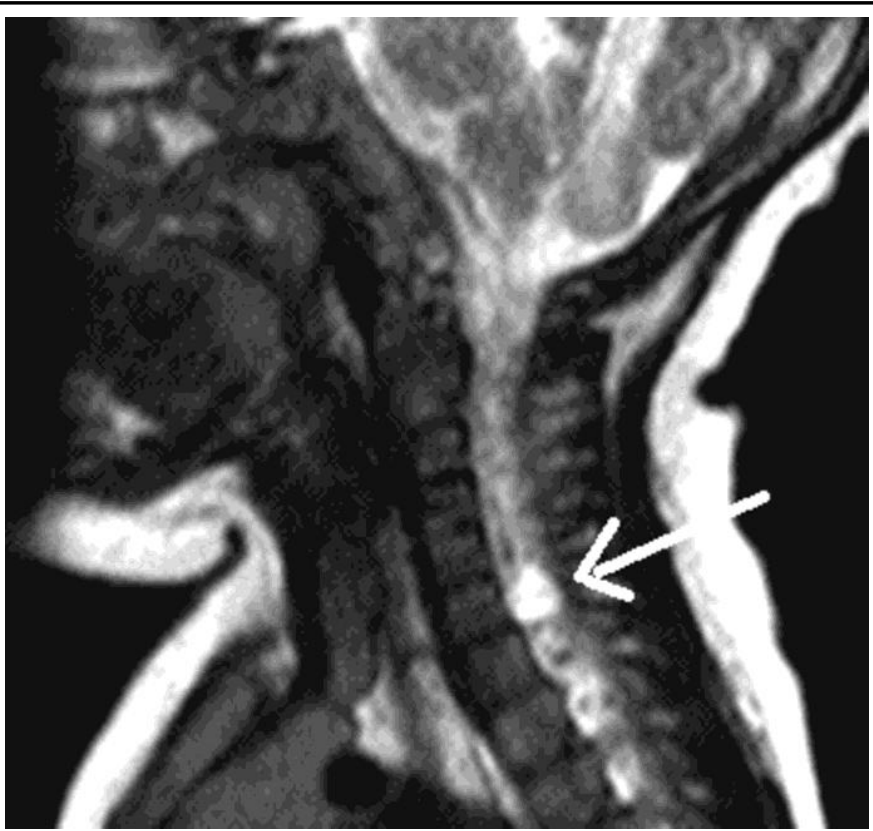

Fig. 8: 5 Month old with suspected left sided brachial plexus palsy .T2 sagittal image shows T2 hyperintensity in at C6-C7 root level suggestive of nerve root avulsion

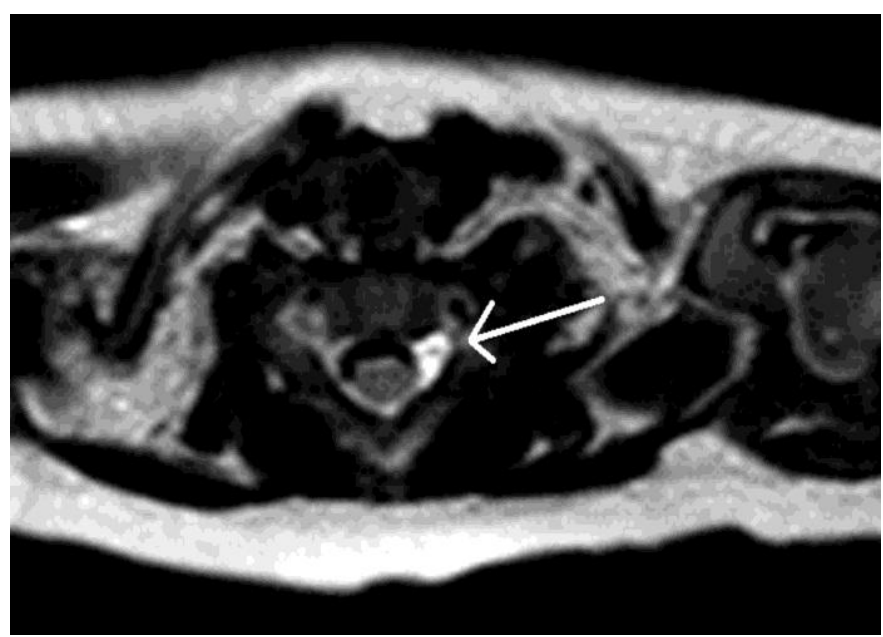

Fig. 9: 5 Month old with suspected left sided brachial plexus palsy .T2 sagittal image shows T2 hyperintensity in at C6-C7 root level suggestive of nerve root avulsion 


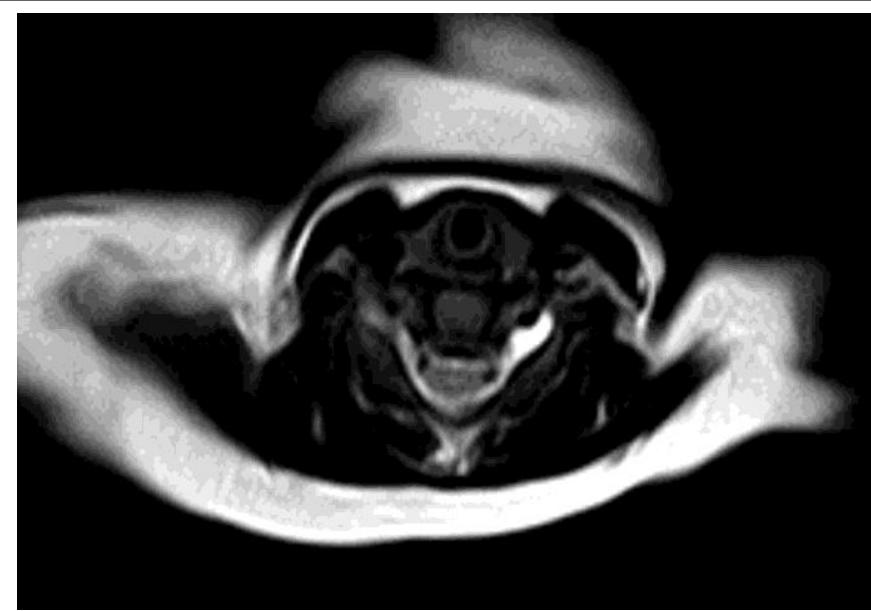

Fig. 10: 10 month old boy with suspected brachial palsy T2 axial images show T2 hyperintense lesions in the trunks of C5-C6 level suggestive of edema and thickening( post ganglionic injury)

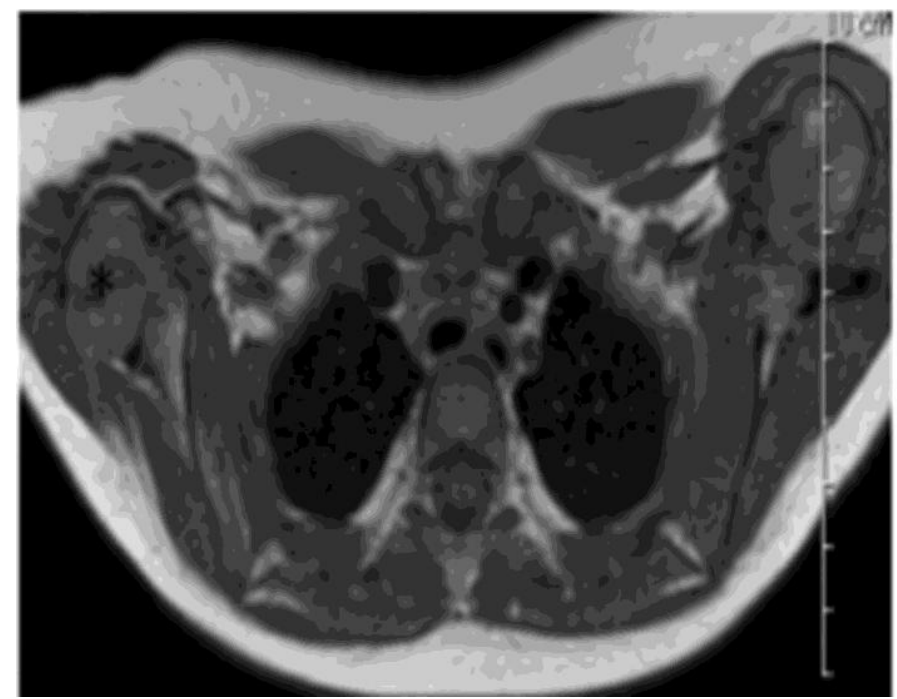

Fig. 11: T1-weighted image demonstrates posterior subluxation of the right humeral head

\section{Conclusion}

MR imaging is an effective tool for demonstrating lesions of the brachial plexus in obstetric birth injuries. The spectrums of findings are vital for further management.

\section{References}

1. Alka Agarwal et al., Role of MRI in Evaluation and Characterization of Brachial Plexopathies Sch. J. App. Med. Sci., August 2015; 3(5C):1949-1953.

2. Somashekar, L.J.S. Yang, M. Ibrahim, and H.A. Parmar High-Resolution MRI Evaluation of Neonatal Brachial Plexus Palsy: A Promising Alternative to Traditional CT Myelography .AJNR Am J Neuroradiology .2014;35:1209 -13.

3. Lawande M. Pictorial essay: role of magnetic resonance imaging in evaluation of brachial plexus pathologies. 2012 Oct Dec. Volume 22 (No 4): p344-349.

4. Baxter D. Tharin, Jonathan A. Kini, Gerald E. York, and John L. Ritter Brachial Plexopathy: A Review of Traumatic and Nontraumatic Causes American Journal of Roentgenology 2014 202:1, W67-W75.

5. Gherman RB, Ouzounian JG, Satin AJ, Goodwin TM, Phelan JP. A comparison of shoulder dystocia-associated transient and permanent brachial plexus palsies. Obstetrics and Gynecology. 2003;102(3):544-548.

6. Yoshikawa T, Hayashi N, Yamamoto S, et al. Brachial plexus injury: clinical manifestations, conventional imaging findings, and the latest imaging techniques. Radiographics 2006;26:S13343. 